\title{
A simple method for coupled acoustic-mechanical analysis with application to gradient-based topology optimization
}

Jensen, Jakob S.

Published in:

Structural and Multidisciplinary Optimization

Link to article, DOI:

10.1007/s00158-018-2147-4

Publication date:

2019

Document Version

Peer reviewed version

Link back to DTU Orbit

Citation (APA):

Jensen, J. S. (2019). A simple method for coupled acoustic-mechanical analysis with application to gradientbased topology optimization. Structural and Multidisciplinary Optimization, 59(5), 1567-1580.

https://doi.org/10.1007/s00158-018-2147-4

\section{General rights}

Copyright and moral rights for the publications made accessible in the public portal are retained by the authors and/or other copyright owners and it is a condition of accessing publications that users recognise and abide by the legal requirements associated with these rights.

- Users may download and print one copy of any publication from the public portal for the purpose of private study or research.

- You may not further distribute the material or use it for any profit-making activity or commercial gain

- You may freely distribute the URL identifying the publication in the public portal

If you believe that this document breaches copyright please contact us providing details, and we will remove access to the work immediately and investigate your claim 


\title{
A simple method for coupled acoustic-mechanical analysis with application to gradient-based topology optimization
}

\author{
Jakob S. Jensen
}

Received: date / Accepted: date

\begin{abstract}
A simple computational framework for analysis of acoustic-mechanical coupling is proposed. The method is based on extended finite element models for structural vibrations and acoustic pressure fluctuations using artificial mechanical and acoustic parameters in the non-structural and non-acoustic domains, respectively. The acoustic-mechanical interaction is created using a self-coupling matrix assembled in the entire computational domain, effectively generating coupling at acoustic-mechanical interface boundaries. The simple analysis tool circumvents the need for explicit interface tracking with accuracy controlled explicitly using a contrast parameter between the physical and artificial material parameters. Furthermore, the method's direct applicability to gradient-based topology optimization where elements can turn from mechanical to acoustic and vice versa, is demonstrated and illustrated using a simple example.
\end{abstract}

\section{Introduction}

The coupling between structural vibrations and air pressure fluctuations plays an important role in many small mechanical components. Microphones rely on air-pressure induced vibrations of thin diaphragms in order to pick up external air-borne signals. Conversely, loudspeakers use the vibrations of the diaphragm to generate air pressure fluctuations perceived by the ear as sound.

In devices such as mobile telephones and hearing aids, microphones, loudspeakers (as well as electrical components such as battery, telecoil, etc.) are packed together in tight space. The miniaturization coupled with the advent of thin, light and flexible materials generate strong coupling and make

\footnotetext{
J.S. Jensen

Department of Mechanical Engineering, Technical University of Denmark, Nils Koppels Allé, Building 404, 2800 Lyngby, Denmark

E-mail: jsj@mek.dtu.dk
}

the acoustic-mechanical performance very sensitive to often minuscule design changes. A major challenge is acoustic feedback where amplified sound from the loudspeaker may be transmitted back to the microphone through a large variety of paths that may be of acoustic, mechanical or coupled type. The feedback loop may result in a disturbing howling noise - a phenomenon which often sets the effective limit of possible signal amplification eg. in hearing aids.

Topology optimization (Bendsøe and Sigmund 2003) is a promising candidate for tackling the feedback problem as well as other design problems linked to the interaction between mechanical vibrations and acoustic pressure fluctuations. In order to correctly assess the dynamics the fully coupled acoustic-mechanical system usually needs to be modelled. This does not only lead to high computational costs but also to challenges in terms of design parameterization. The analysis model encompasses domains with both structural and acoustic elements and in order to enforce the coupling conditions the interface boundaries between domains have to tracked explicitly. This requirement conflicts with the nature of topology optimization with a pixel (or voxel)based design description where structural-acoustic interfaces may appear or disappear ad-hoc during the optimization procedure.

Thus, there appears to be a strong need for developing modelling schemes that allow for a seamless transition between acoustical and structural domains. This challenging parametrization problem has been treated previously using different methods. In Yoon et al (2007) a mixed finite element scheme was suggested to circumvent the need for explicit interface tracking and was used to formulate a corresponding topology optimization scheme. In this formulation the pressure is introduced in the structural model as an auxiliary variable by splitting the stress into its volumetric and deviatoric part. The resulting model can be used to simulate both structural vibrations and acoustic pressure fluctuations 
with an appropriate choice of system parameters (Wang and Bathe 1997). In this way it is possible to directly interpolate between a structural and an acoustic medium (with vanishing shear modulus) using a single design variable. This formulation was inspired by the earlier work by Sigmund and Clausen (2007) where the approach was applied to deal with topology optimization of a static structural problem with design dependent pressure load. The method has also later been used by Kook and Jensen (2017) to optimize acousticmechanical microstructures wrt. damping properties. An alternative to the mixed finite element approach is found in the adaptation of the level set method to the acoustic-mechanical coupling problem. The level set method was originally developed by Osher and Sethian (1988) for simulation of front propagation and was introduced for design optimization by Sethian and Wiegmann (2000), Osher and Santosa (2001) and further developed by Allaire et al (2002) and Wang et al (2003). In Shu et al (2014) the level set method was adapted to acoustic-mechanical problems providing explicit knowledge of the boundary location and was recently applied by Desai et al (2018) for both pure acoustic and coupled problems. Another approach adapts a unified multiphase (UMP) model based on Biot theory for porous media which was presented in Lee et al (2012) and used for topology optimization using the density approach in Lee et al (2015) and using the level set approach in Noguchi et al (2015). Similarly to the mixed formulation the coupling between the acoustic and structural domains are inherent in the formulation and the coupling interface needs not to be explicitly tracked during the optimization procedure. Further development of the method using a level set parametrization can be found in Noguchi et al (2016), Noguchi et al (2017) and was recently used by Miyata et al (2018) to design an acoustic metasurface to convert acoustic waves into elastic transverse waves.

In Søndergaard and Pedersen (2014) a topology optimization problem was considered for the suspension of the loudspeaker (receiver) in a hearing aid device. However, here the inherent parametrization difficulties were circumvented by specifying the design domain so that structural elements could be replaced by void. Other workarounds for facilitating topology optimization of acoustic-structural problems have been presented previously. In Vicente et al (2015) a standard parametrization and corresponding sensitivity analysis valid for solid-void models was used but the void heuristically replaced by an acoustic medium. In the paper the design updates were based on the BESO method rather than a SIMP model and mathematical programming tools. The BESO method has recently also been combined with the mixed finite element method in Kook (2017).

Other works have considered topology optimization for acoustic-structural interaction problems where the parametrization does not lead to a reorganization of structural and acous- tic domains which simplifies the problem considerably. Akl et al (2009) considered the coupled problem of an elastic plate and an acoustic cavity and optimized the thickness distribution of the plate in order to minimize the interaction effect. Du and Olhoff (2010) considered optimization of a bi-material plate in order to optimize the acoustic radiation. In this case a simplified model for radiation was developed for weak coupling neglecting the fully coupled effects. In Chen et al (2017) the bi-material plate problem was studied again using the fully coupled interaction model in combination with an optimality criteria condition. These topology optimization studies were preceded by many other studies considering design optimization for vibro-acoustic response. For an overview of earlier optimization work see eg. Marburg (2002).

The present paper presents a new simple method for analyzing acoustic-mechanical coupling and its application to gradient-based topology optimization is proposed. Unlike methods based on the mixed formulation and the UMP model, to which the proposed method bears some resemblance, it involves only standard finite element formulations for the acoustic and structural problems and applies explicit coupling matrices between the two fields.

The paper is organized as follows. The analysis model is formulated in the next section considering a well defined division of structural and acoustic domains. The accuracy of the method is demonstrated using simple test cases. In the final part of the paper the method is extended to gradientbased topology optimization and is illustrated with a test example.

\section{FE model}

The paper considers the general analysis problem illustrated in Fig. 1. A mechanical structure with given boundary conditions is excited by a time-dependent force $f(t)$. The solid structure is surrounded by an external acoustic medium and may posses internal acoustic cavities as well. Furthermore, we may consider time-dependent acoustic excitation given as $p(t)$.

The main goal here is to develop a formulation for the analysis of acoustic-structural coupling that circumvents the need for explicit tracking of the interface boundaries and facilitates a transition from solid to acoustic domains and vice versa. Of special consideration is to create a model that can be applied to gradient-based topology optimization. In Fig. 2 we show an example of an acoustic-structural interaction problem where the part of the domain occupied by solid is denoted $\Omega_{s}$ and the part occupied by the acoustic medium is denoted $\Omega_{a}$.

A fully-coupled finite element model for time-harmonic structural vibrations and pressure fluctuations is now constructed. The computational domain $\Omega_{s} \cap \Omega_{a}$ is discretized 


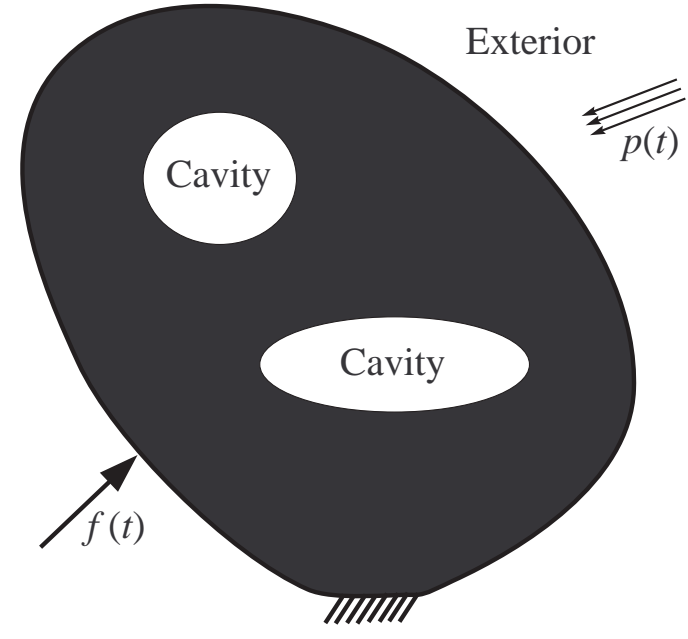

Fig. 1 General analysis problem for a coupled acoustic-mechanical system.

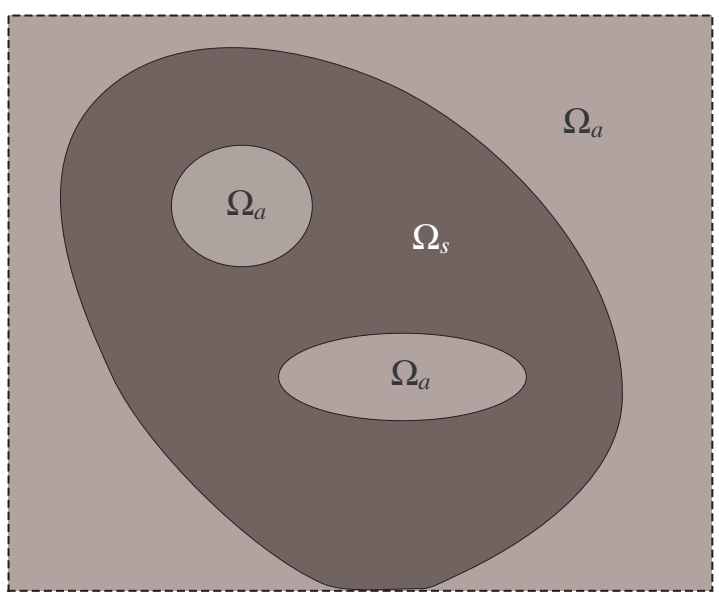

Fig. 2 Identification of acoustic domain $\Omega_{a}$ and solid domains $\Omega_{s}$.

into a number, ne, of suitable elements and a discrete element topological indicator $\chi^{e}$ is introduced in order to identify the two parts of domains:

$\chi^{e}=\left\{\begin{array}{l}1 \text { if } e \in \Omega_{s} \\ 0 \text { if } e \in \Omega_{a}\end{array}\right.$

thus, for $\chi^{e}=1$ we have a solid and for $\chi^{e}=0$ we have an acoustic part of the domain. The reason to choose a discrete topology indicator in this part of the paper is to simplify the description as much as possible. In a later section the discrete indicator $\chi^{e}$ will be replaced by a continuous indicator $\xi^{e}$. It should be emphasized that there is no restriction to the choice of element geometry. However, since the main goal of the proposed method is to allow for a change of topology, it is most natural to choose a regular grid with the same element geometry in the acoustic and solid domains. This is well knowing that, depending on the material properties of the solid, the discretization necessary to obtain an accurate solution in the acoustic and solid domain may be significantly different.

The proposed scheme involves both a structural and an acoustic model defined on the entire computational domain to be designed. This naturally increases the computational burden compared to standard acoustic-structural simulation methods but allows for the construction of a simple algorithm directly applicable to the topology optimization procedure.

\subsection{Structural vibrations}

We will now set up the basic finite element model for structural vibrations of the solid part of the structure. Fig. 3 illustrates the material properties used for the solid model, where we use $\rho_{s}$ and $E$ to represent the mass density and Young's modulus of the solid, respectively. Poisson's ratio of the solid is given as $v$. The structural domain is extended with the part of the domain occupied by the acoustic medium. In this part we introduce the artificial material parameters $\rho_{v}$ and $E_{v}$ to indicate the properties corresponding to a structural void (Poisson's ratio is kept constant). These values should naturally be chosen such that the effect of including the extra non-solid domain in the model is minimal. It should noted here that the approach followed here is completely analogous to the approach taken in standard density based topology optimization where regions of void with artificial material parameters are also included in the structural model.

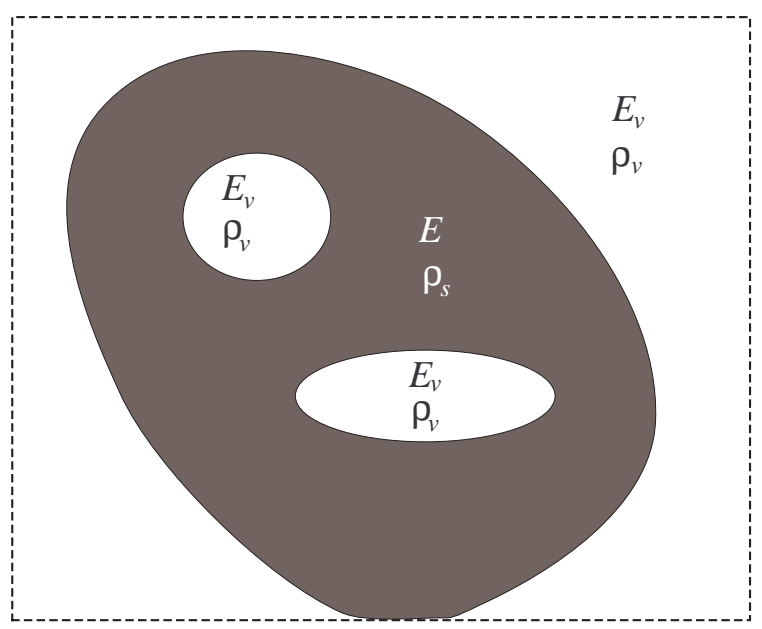

Fig. 3 Material properties for the solid modelling encompassing properties in the actual solid domain and in the extended part of the domain occupied by the acoustic medium.

The finite element model for time-harmonic structural vibrations is now set up using a standard Galerkin method 
in the entire domain $\Omega$ :

$\left(-\omega^{2} \mathbf{M}_{s}+\mathbf{K}_{s}\right) \mathbf{u}=\mathbf{f}_{s}$

where $\mathbf{u}$ is a vector of nodal vibration amplitudes, $\omega$ is the frequency of vibration, $\mathbf{f}_{s}$ is the structural excitation vector assembled in a usual manner, and the mass and stiffness matrices are specified as:

$$
\begin{aligned}
\mathbf{M}_{s} & =\sum_{e=1}^{n e} \rho_{s}^{e} \mathbf{M}^{e} \\
\mathbf{K}_{s} & =\sum_{e=1}^{n e} E^{e} \mathbf{K}_{s}^{e}
\end{aligned}
$$

in which $\rho_{s}^{e}$ and $E^{e}$ are the element values of the structural mass density and the Young's modulus, respectively.

The element values are specified in terms of the topological indicator $\chi^{e}$ as:

$\rho_{s}^{e}, E^{e}= \begin{cases}\rho_{s}, E & \text { if } \chi^{e}=1 \\ \rho_{v}, E_{v} & \text { if } \chi^{e}=0\end{cases}$

and the element matrices are specified as:

$$
\begin{aligned}
\mathbf{M}_{s}^{e} & =\int_{\Omega^{e}} \mathbf{N}_{s}^{\mathrm{T}} \mathbf{N}_{s} \mathrm{~d} \Omega^{e} \\
\mathbf{K}_{s}^{e} & =\int_{\Omega^{e}} \mathbf{B}_{s}^{\mathrm{T}} \tilde{\mathbf{C}} \mathbf{B}_{s} \mathrm{~d} \Omega^{e}
\end{aligned}
$$

in which $\mathbf{N}_{s}$ and $\mathbf{B}_{s}$ are the structural shape function and strain-displacement matrices and $\tilde{\mathbf{C}}$ is the constitutive matrix with Young's modulus set to unity.

Structural damping has been omitted for simplicity but can be included in a straightforward manner.

\subsection{Acoustic pressure fluctuations}

The finite element model for the acoustic pressure fluctuation is set up in a similar way in the full domain encompassing also the part of the domain occupied by the solid material. Fig. 4 shows the material properties used with $\rho_{a}$ representing the mass density of the acoustic medium and $\kappa$ being the bulk modulus given as $\kappa=\rho_{a} c^{2}$, with $c$ being the speed of sound in air.

The finite element model for the acoustic pressure oscillations then becomes:

$\left(-\omega^{2} \mathbf{M}_{a}+\mathbf{K}_{a}\right) \mathbf{p}=\mathbf{f}_{a}$

in which $\mathbf{p}$ contains the nodal pressure amplitudes, $\mathbf{f}_{a}$ is the acoustic excitation vector and the matrices are specified as:

$$
\begin{aligned}
\mathbf{M}_{a} & =\sum_{e=1}^{n e} \frac{1}{\kappa^{e}} \mathbf{M}_{a}^{e} \\
\mathbf{K}_{a} & =\sum_{e=1}^{n e} \frac{1}{\rho_{a}^{e}} \mathbf{K}_{a}^{e}
\end{aligned}
$$

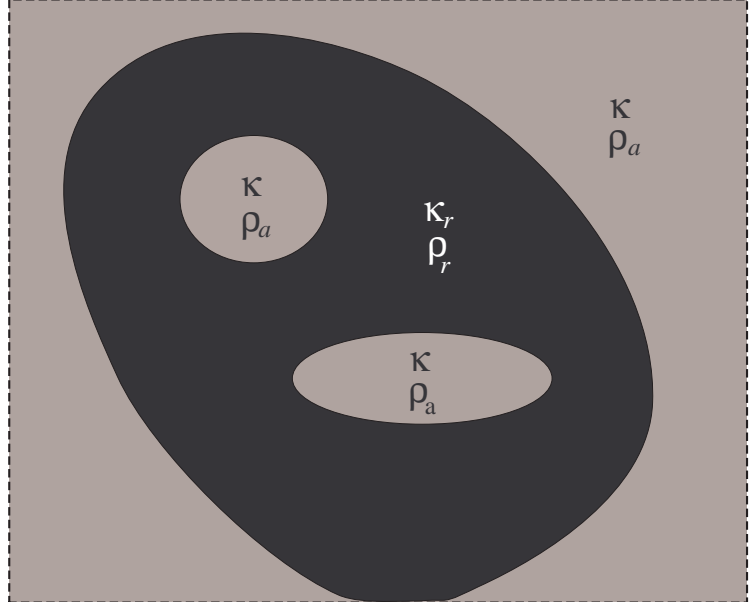

Fig. 4 Material properties for the acoustic model encompassing properties in the acoustic domain and in the extended part of the domain occupied by the solid medium.

with $\rho_{a}^{e}$ being the element mass density and $\kappa^{e}$ is the element bulk modulus of the acoustic medium.

The element-wise properties are for the acoustic problem defined in terms of the topological indicator $\chi^{e}$ as:

$\rho_{a}^{e}, \kappa^{e}=\left\{\begin{array}{l}\rho_{a}, \kappa \text { if } \chi^{e}=0 \\ \rho_{r}, \kappa_{r} \text { if } \chi^{e}=1\end{array}\right.$

where $\rho_{r}, \kappa_{r}$ are the artificial values used to represent the non-acoustic (rigid) domain. Again these values should be chosen to minimize the effect of including the non-acoustic domain in the acoustic model.

The element matrices are here given as:

$$
\begin{aligned}
\mathbf{M}_{a}^{e} & =\int_{\Omega^{e}} \mathbf{N}_{a}^{\mathrm{T}} \mathbf{N}_{a} \mathrm{~d} \Omega^{e} \\
\mathbf{K}_{a}^{e} & =\int_{\Omega^{e}} \mathbf{B}_{a}^{\mathrm{T}} \mathbf{B}_{a} \mathrm{~d} \Omega^{e}
\end{aligned}
$$

where $\mathbf{N}_{a}$ is a vector of acoustic shape function and $\mathbf{B}_{a}$ is a matrix with the corresponding spatial derivatives.

Normal boundary conditions such as prescribed displacement and pressure are enforced for both structural and acoustic problems in the usual manner. Other boundary conditions can be applied using standard formulations, e.g. absorbing acoustic boundaries leading to an extra element damping matrix:

$\mathbf{C}_{a}^{e}=\int_{\Gamma^{e}} \mathbf{N}_{a}^{\mathrm{T}} \mathbf{N}_{a} \mathrm{~d} \Gamma^{e}$

integrated along the absorbing element boundary and assembled into a global damping matrix as

$\mathbf{C}_{a}=\sum_{e=1}^{n e^{*}} \frac{1}{\sqrt{\rho_{a}^{e} \kappa_{a}^{e}}} \mathbf{C}_{a}^{e}$

where $n e^{*}$ indicates elements along the absorbing boundary. Additionally, more advanced models such as perfectly 


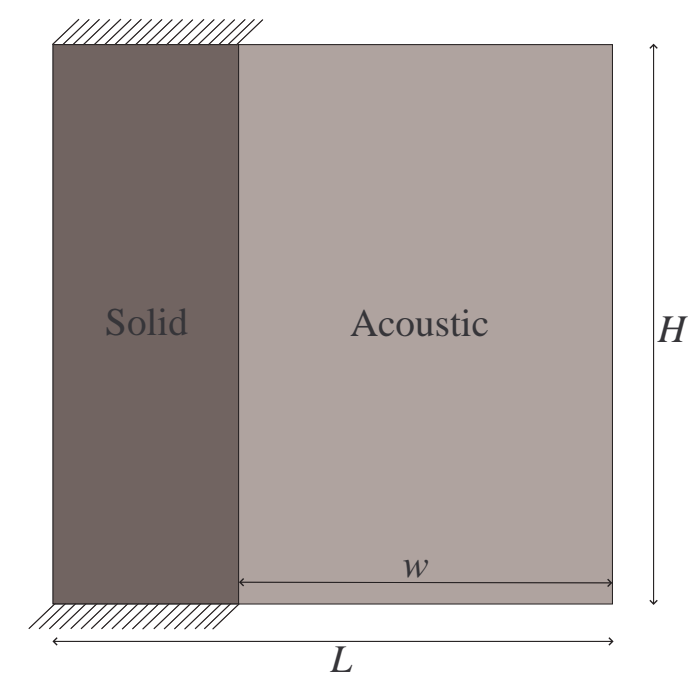

Fig. 5 Test case used to study the effect of the contrast parameter $\beta$ on structural and acoustic eigenvalues.

matched layers (PML) can be applied in the standard way without any modifications.

We refer to standard textbooks, such as eg. Cook et al (2002), for more details on finite element analysis of structural and acoustic problems.

\subsection{Effect of artificial material parameters}

The choice of the artificial parameters affects the accuracy of the computations for both the structural vibrations and the acoustic pressure. To study the accuracy of the extended domain approach, a one-parameter contrast model is introduced and tested using a simple example where the eigenvalues of a simple solid structure and an adjacent acoustic cavity are computed.

The setup for the test case is illustrated in Fig. 5. A square domain is divided into a solid part filling one-third of the domain and with the remaining part of the domain being filled by an acoustic domain. The structure is fixed in the upper and lower part and the acoustic domain has hard-wall boundary conditions at the top and rightmost boundaries. Structural and acoustic eigenvalues are computed on the extended domains and compared to reference values computed by assembling the corresponding problems on the structural and acoustic parts only. The geometrical and material parameters used are listed in Table 1.

The following empirical contrast model is proposed:

$\frac{\rho_{s}}{\rho_{v}}=\frac{\kappa_{r}}{\kappa}=10^{\beta}$

$\frac{E}{E_{v}}=\frac{\rho_{r}}{\rho_{a}}=10^{\beta-1}$

\begin{tabular}{|l|r|}
\hline$E$ & $500 \mathrm{MPa}$ \\
$\rho_{s}$ & $1200 \mathrm{~kg} / \mathrm{m}^{3}$ \\
$v$ & 0.3 \\
$2 \mathrm{D}$ model & plane strain \\
$\rho_{a}$ & $1.204 \mathrm{~kg} / \mathrm{m}^{3}$ \\
$c$ & $342 \mathrm{~m} / \mathrm{s}$ \\
$H$ & $1.0 \mathrm{~m}$ \\
$L$ & $1.0 \mathrm{~m}$ \\
$w$ & $1 / 3 \mathrm{~m}$ \\
mesh & $50 \times 50$ \\
\hline
\end{tabular}

Table 1 Geometrical, material and mesh parameters for the eigenvalue test example in Fig. 5.

where $\beta$ is a parameter controlling the absolute contrast of material parameters. It was found here that using a contrast one order of magnitude larger for the mass density than for Young's modulus and similarly one order of magnitude larger for bulk modulus than for the mass density of the acoustic medium produces the best results without any spurious modes in the extended domains. A high value of $\beta$ is naturally expected to improve the analysis accuracy which is validated in Fig. 6, where the relative error of the extended domain computations is illustrated for the first six structural and acoustic modes. It is noted that for all modes computed a nice convergence towards the reference values is obtained with increasing $\beta$. In Figs. 7-8 selected structural and acoustic modes are shown for $\beta=5$. For both displacement and acoustic pressure fields we note smooth decaying behavior within the extended domains.

However, it should be mentioned that limitations of the use of simple constrast models in combination with standard finite element procedures for the acoustic problem has been pointed out by Kasolis et al (2015). Here it was reported to lead to spurious resonances inside the rigid domains and ill-conditioned matrices and a stabilization scheme was proposed to remedy the problems. However, for the examples considered in this work these issues have not been observed and the use of more advanced models are left for future work.

\section{Acoustic-structural coupling}

The previous sections involved an extension of the structural and acoustic domains to the full model domain. This is done to allow for structural parts to be able to turn into acoustic domains and vice versa. What remains now is to compute the coupling between the structural vibrations and the pressure fluctuation in a way so that the location of the interface does not need to be explicitly specified. 

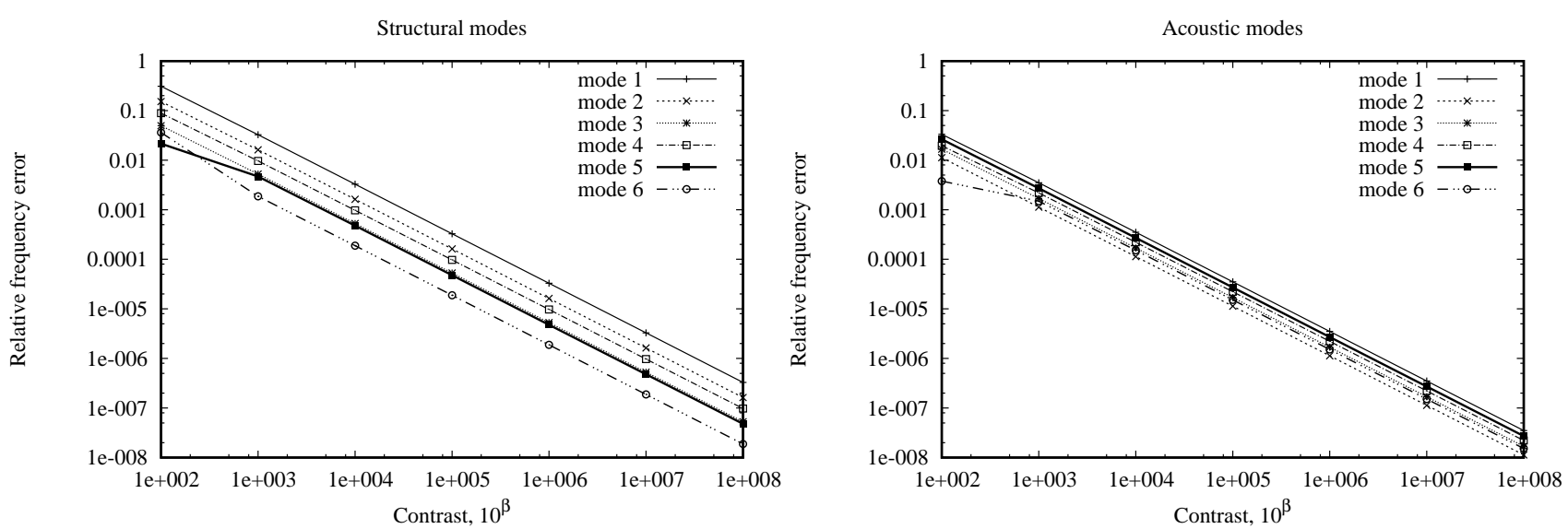

Fig. 6 Relative error of structural and acoustical eigenvalues by using the extended domain with artificial material parameters. Error shown vs contrast measure $10^{\beta}$

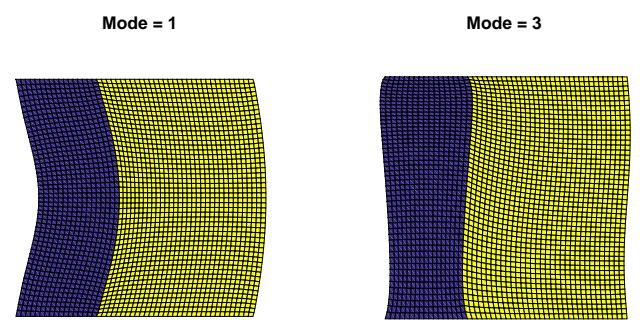

Fig. 7 Two selected structural mode shapes computed with the extended domain $(\beta=5)$.
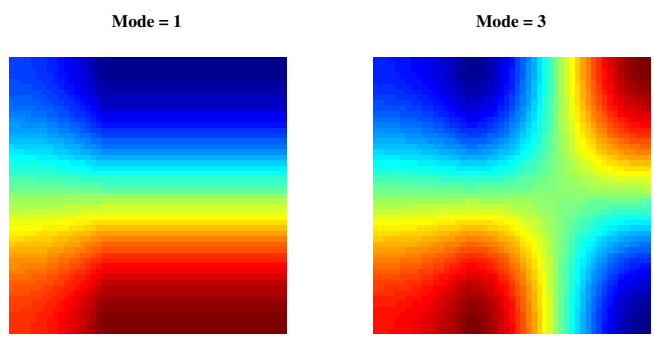

Fig. 8 Two selected acoustical mode shapes computed with the extended domain $(\beta=5)$.

\subsection{The conventional approach}

The coupling between the acoustic pressure field and the structural vibrations acts in a two-way fashion. The acceleration of the vibrating structural boundary acts as an acoustic source emitting pressure waves into the acoustic domain. Conversely, the acoustic pressure wave acts as a boundary load on the structural domain. We may express these equivalent structural loads and acoustic sources as

$\mathbf{f}_{s}^{p}=\mathbf{S}^{\mathrm{T}} \mathbf{p}$

$\mathbf{f}_{a}^{u}=\omega^{2} \mathbf{S u}$

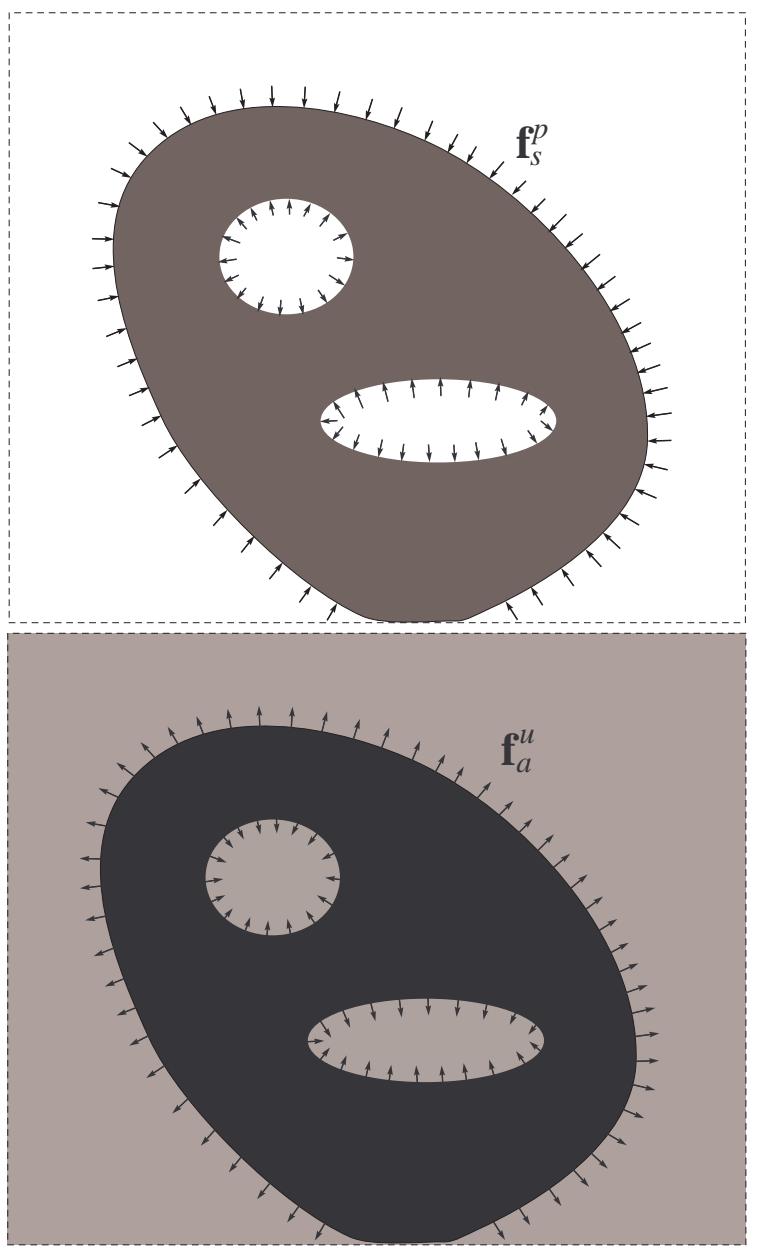

Fig. 9 Illustration of the structural loads coming from the acoustic pressure field and the acoustic sources coming from the structural vibrations of the solid.

and these are illustrated in Fig. 9. 
The matrix $\mathbf{S}$ is the coupling matrix found in the conventional approach as

$$
\begin{aligned}
\mathbf{S} & =\sum_{e=1}^{n e i n t} \mathbf{S}^{e} \\
\mathbf{S}^{e} & =\int_{\Gamma_{\text {int }}}\left(\mathbf{n}_{a}^{\mathrm{T}} \mathbf{N}_{s}\right)^{\mathrm{T}} \mathbf{N}_{a} \mathrm{~d} \Gamma
\end{aligned}
$$

where the shape functions for the structure and for the acoustic domain are evaluated for the structural and acoustic elements at the coupling interface and $\mathbf{n}_{a}$ is the normal vector pointing from the acoustic domain into the structural domain. The assembly is performed only for element pairs along the interface boundary, thus, in order to compute the integral we must know the location of the interface and identify the involved elements. See eg. Cook et al (2002) for more details.

\subsection{Topological indicator-based coupling matrix}

In this section we develop an alternative computation of $\mathbf{f}_{s}^{p}$ and $\mathbf{f}_{a}^{u}$ based on the element indicator $\chi^{e}$. The key to the proposed algorithm is the computation of an element selfcoupling matrix.

The self-coupling matrix is computed in a similar way as when computing the coupling matrix in the traditional approach - however the acoustic and structural shape functions are now evaluated for the same element and integrated over all boundaries. We define this self-interaction matrix as

$\mathbf{S}^{e}=\oint_{\Gamma}\left(\mathbf{n}^{\mathrm{T}} \mathbf{N}_{s}\right)^{\mathrm{T}} \mathbf{N}_{a} \mathrm{~d} \Gamma$

where $\mathbf{n}$ is the outward pointing normal vector from the element boundary. In the appendix the computation of $\mathbf{S}^{e}$ is exemplified for the bilinear rectangular 4-noded element (Q4). The self-coupling matrix simply expresses the intrinsic coupling between the nodal loads and pressures for a given element.

In order to assemble the global coupling matrices we loop over all elements in the following manner:

$\mathbf{S}_{p}=\sum_{e=1}^{n e}\left(1-\chi^{e}\right) \mathbf{S}^{e}$

$\mathbf{S}_{u}=\sum_{e=1}^{n e} \chi^{e}\left(\mathbf{S}^{e}\right)^{\mathrm{T}}$

It should be noted that since we multiply element contributions by factors $\left(1-\chi^{e}\right)$ and $\chi^{e}$, we include only pressureinduced structural loads from acoustic elements and vibrationinduced acoustic sources from solid elements.

In the assembly procedure contributions to $\mathbf{S}_{p}$ in the interior part of the acoustic domain vanish since contributions from neighboring elements cancel out. The same holds true for contributions to $\mathbf{S}_{u}$ in the interior part of the solid domain. What is left are sparse matrices that represent the coupling at the acoustic-mechanical interface in addition to non-vanishing contributions at the exterior domain boundary which can be removed by standard boundary condition operations. In fact, in the discrete case where $\chi^{e}$ takes only 0 and 1 values, and after enforcing appropriate boundary conditions on the two coupling matrices, these will in fact obey the relation $\mathbf{S}_{p}=\mathbf{S}_{u}^{\mathrm{T}}$ and be identical to the coupling matrix obtained using the traditional approach. However, when we later extend the approach to allow for continuous values of the topological indicator function, the two matrices will in general be different. It should be emphasized that even though the coupling matrices in the discrete case reduce to the standard coupling matrix, the structural and acoustic problems are still solved on the full extended domains. Hence the discrete problem is not equivalent to a standard segregated analysis procedure.

We can now define the global load and source vectors

$$
\mathbf{f}_{s}^{p}=\mathbf{S}_{p} \mathbf{p}
$$

$\mathbf{f}_{a}^{u}=\omega^{2} \mathbf{S}_{u} \mathbf{u}$

and set up the coupled equations for the acoustic-structural interaction problem:

$$
\begin{aligned}
& \left(-\omega^{2} \mathbf{M}_{s}+\mathbf{K}_{s}\right) \mathbf{u}=\mathbf{f}_{s}+\mathbf{S}_{p} \mathbf{p} \\
& \left(-\omega^{2} \mathbf{M}_{a}+\mathbf{K}_{a}\right) \mathbf{p}=\mathbf{f}_{a}+\omega^{2} \mathbf{S}_{u} \mathbf{u}
\end{aligned}
$$

which we can convert into:

$$
\left(\left[\begin{array}{cc}
\mathbf{K}_{s} & \mathbf{S}_{p} \\
\mathbf{0} & \mathbf{K}_{a}
\end{array}\right]-\omega^{2}\left[\begin{array}{cc}
\mathbf{M}_{s} & \mathbf{0} \\
\mathbf{S}_{u} & \mathbf{M}_{a}
\end{array}\right]\right)\left\{\begin{array}{l}
\mathbf{u} \\
\mathbf{p}
\end{array}\right\}=\left\{\begin{array}{l}
\mathbf{f}_{s} \\
\mathbf{f}_{a}
\end{array}\right\}
$$

which we can also write in compact notation as:

$$
\left(\tilde{\mathbf{K}}-\omega^{2} \tilde{\mathbf{M}}\right) \tilde{\mathbf{u}}=\tilde{\mathbf{S}} \tilde{\mathbf{u}}=\tilde{\mathbf{f}}
$$

in which $\tilde{\mathbf{S}}$ has been introduced as the dynamic system matrix.

\subsection{Example: Acoustic transmission through a flexible partition}

We now demonstrate the method when applied to the problem of transmission of a plane acoustic wave through a flexible partition. Furthermore, an inherent limitation of the developed model, wrt. to the use of the contrast parameter $\beta$, is highlighted.

Fig. 10 shows the model used for the test case. A timeharmonic plane acoustic wave of unit amplitude impinges on a flexible partition with fixed boundary conditions specified on the top and bottom part. The induced structural vibrations of the partition cause emission of sound waves in the acoustic domain to the right and the transmission is recorded as $|p|^{2}$ averaged on the right boundary. In the acoustic parts the top and bottom boundaries are specified as sound hard 


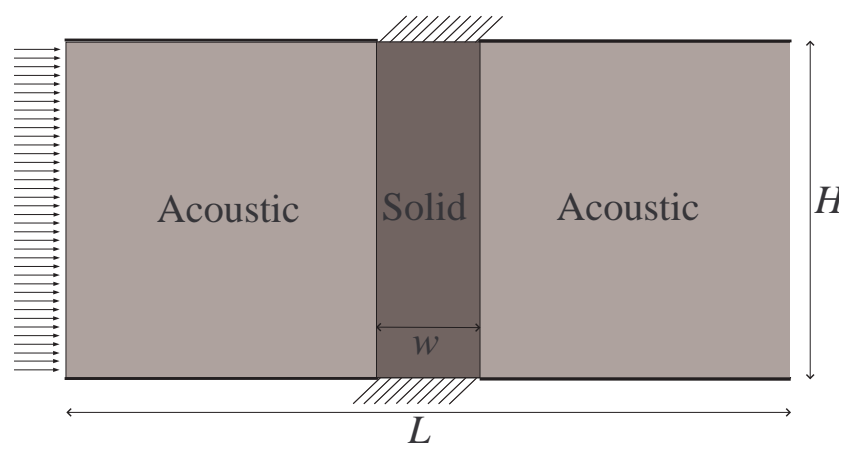

Fig. 10 Test case for simulating the acoustic transmission of a timeharmonic plane wave through a flexible partition.

\begin{tabular}{|l|r|}
\hline$E$ & $500 \mathrm{kPa}$ \\
$\rho_{s}$ & $1000 \mathrm{~kg} / \mathrm{m}^{3}$ \\
$\nu$ & 0.3 \\
$2 \mathrm{D}$ model & plane strain \\
$\rho_{a}$ & $1.204 \mathrm{~kg} / \mathrm{m}^{3}$ \\
$c$ & $342 \mathrm{~m} / \mathrm{s}$ \\
$H$ & $0.4 \mathrm{~m}$ \\
$L$ & $1.0 \mathrm{~m}$ \\
$w$ & $0.05 \mathrm{~m}$ \\
mesh & $200 \times 80$ \\
\hline
\end{tabular}

Table 2 Geometrical, material and mesh parameters for acoustic transmission example.

and absorbing boundaries are specified on the left and right boundary. The geometrical and material parameters used are listed in Table 2. It should be noted that a very soft material is used merely to broaden the resonance peak in the transmission spectrum, that would otherwise be very sharp.

Fig. 11 shows the transmission in the frequency range from 0 to $10[\mathrm{~Hz}]$ encompassing the fundamental symmetric mode of vibration for the chosen geometrical dimensions and material parameters. Full transmission is reached near the natural frequency of the fundamental mode and the transmission drops away from the resonance frequency. The effect of the choice of the contrast parameter $\beta$ is clearly observed in the figure. As was noted in the eigenvalue test case, the acoustic field does not vanish in the extended domain but displays a smooth decaying behavior away from the acoustic-mechanical interface. For this test case it also implies that if $\beta$ is too small, a non-negligible transmission is obtained at the lower frequencies due to acoustic leakage through the solid domain. This is a parasitic effect and not desirable, but the effect can effectively be controlled by choosing a higher value of $\beta$. For the specific example, choosing $\beta$ to 8 or more will result in a transmission spectrum indistinguishable from the reference COMSOL results.

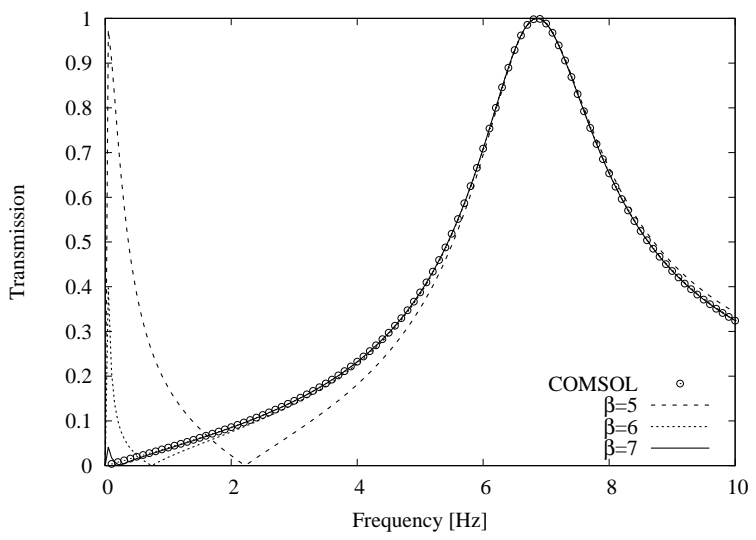

Fig. 11 The acoustic transmission computed using the proposed method for different values of the contrast parameter $\beta$. Results compared to reference results obtained using COMSOL with identical mesh and shape functions.

\section{Application to topology optimization}

In order to apply the developed formulation to a gradientbased topology optimization scheme, the discrete element indicator function $\chi^{e}$ is replaced by a continuous indicator function:

$\chi^{e} \rightarrow \xi^{e}$

where $\xi^{e}$ takes values between 0 and 1 . The formulation derived in the previous sections can directly be used also for continuous values of the indicator function. The two coupling matrices will now in general be fully populated corresponding to induced structural loads and acoustic sources distributed in the entire domain. This should be seen only as an intermediate step towards reaching the discrete (close to) 0 and 1 values of the indicator function in the final optimized structures. It should be emphasized that due to the timeharmonic formulation the appearance of loads and sources distributed in the domain do not pose problems wrt. wellposedness of the state problem. This is in contrast to a similar formulation prosed for electro-statically induced structural loads proposed by Yoon and Sigmund (2008) where distributed loads could results in singular static structural problems and had to be eliminated.

The derived formulation allows us to derive the gradient of a generally formulated objective function wrt. our continuous topological indicator function - which we will use as our design variable. We will consider a real-valued objective function of the following form:

$\Phi=c\left(\boldsymbol{\xi}, \tilde{\mathbf{u}}_{r}, \tilde{\mathbf{u}}_{i}\right)$

where we explicitly specify the dependency of the objective function on the real and imaginary parts of the state vector $\tilde{\mathbf{u}}$ that comprise both the vibration amplitude field $\mathbf{u}$ and the acoustic pressure field $\mathbf{p}$. The objective function may also depend explicitly on the design variable vector $\boldsymbol{\xi}$. 
We now find the gradient wrt. a single design variable $\xi^{e}$ as using the chain rule:

$\Phi^{\prime}=\frac{d \Phi}{d \xi^{e}}=\frac{\partial c}{\partial \xi^{e}}+\frac{\partial c}{\partial \tilde{\mathbf{u}}_{r}} \frac{\partial \tilde{\mathbf{u}}_{r}}{\partial \xi^{e}}+\frac{\partial c}{\partial \tilde{\mathbf{u}}_{i}} \frac{\partial \tilde{\mathbf{u}}_{i}}{\partial \xi^{e}}$

The adjoint approach will be employed to ensure efficient computation of $\Phi^{\prime}$ also for a large number of design variables. The approach employs the augmented objective function:

$\Phi=c+\boldsymbol{\lambda}_{r}^{\mathrm{T}} \mathbf{r}_{r}-\boldsymbol{\lambda}_{i}^{\mathrm{T}} \mathbf{r}_{i}$

which is based on a complex adjoint field vector $\boldsymbol{\lambda}=\boldsymbol{\lambda}_{r}+$ $i \boldsymbol{\lambda}_{i}$ and the residual of the state equation $\mathbf{r}=\tilde{\mathbf{S}} \tilde{\mathbf{u}}-\tilde{\mathbf{f}}=\mathbf{0}$, here split up into its real and imaginary parts as:

$\mathbf{r}_{r}=\tilde{\mathbf{S}}_{r} \tilde{\mathbf{u}}_{r}-\tilde{\mathbf{S}}_{i} \tilde{\mathbf{u}}_{i}-\tilde{\mathbf{f}}_{r}=\mathbf{0}$

$\mathbf{r}_{i}=\tilde{\mathbf{S}}_{i} \tilde{\mathbf{u}}_{r}+\tilde{\mathbf{S}}_{r} \tilde{\mathbf{u}}_{i}-\tilde{\mathbf{f}}_{i}=\mathbf{0}$

The augmented expression for the gradient $\Phi^{\prime}$ now becomes:

$$
\begin{aligned}
\Phi^{\prime} & =\frac{\partial c}{\partial \xi^{e}}+\frac{\partial c}{\partial \tilde{\mathbf{u}}_{r}} \frac{\partial \tilde{\mathbf{u}}_{r}}{\partial \xi^{e}}+\frac{\partial c}{\partial \tilde{\mathbf{u}}_{i}} \frac{\partial \tilde{\mathbf{u}}_{i}}{\partial \xi^{e}} \\
& +\boldsymbol{\lambda}_{r}^{\mathrm{T}}\left(\tilde{\mathbf{S}}_{r} \frac{\partial \tilde{\mathbf{u}}_{r}}{\partial \xi^{e}}+\frac{\partial \tilde{\mathbf{S}}_{r}}{\partial \xi^{e}} \tilde{\mathbf{u}}_{r}-\tilde{\mathbf{S}}_{i} \frac{\partial \tilde{\mathbf{u}}_{i}}{\partial \xi^{e}}-\frac{\partial \tilde{\mathbf{S}}_{i}}{\partial \xi^{e}} \tilde{\mathbf{u}}_{i}-\frac{\partial \tilde{\mathbf{f}}_{r}}{\partial \xi^{e}}\right) \\
& -\boldsymbol{\lambda}_{i}^{\mathrm{T}}\left(\tilde{\mathbf{S}}_{i} \frac{\partial \tilde{\mathbf{u}}_{r}}{\partial \xi^{e}}+\frac{\partial \tilde{\mathbf{S}}_{i}}{\partial \xi^{e}} \tilde{\mathbf{u}}_{r}+\tilde{\mathbf{S}}_{r} \frac{\partial \tilde{\mathbf{u}}_{i}}{\partial \xi^{e}}+\frac{\partial \tilde{\mathbf{S}}_{r}}{\partial \xi^{e}} \tilde{\mathbf{u}}_{i}-\frac{\partial \tilde{\mathbf{f}}_{i}}{\partial \xi^{e}}\right)
\end{aligned}
$$

After collecting terms that involve $\frac{\partial \tilde{\mathbf{u}}_{r}}{\partial \xi^{e}}$ and $\frac{\partial \tilde{\mathbf{u}}_{i}}{\partial \xi^{e}}$ (assuming that external forces and acoustic sources are independent of the design) we obtain:

$$
\begin{aligned}
& \Phi^{\prime}=\frac{\partial c}{\partial \xi^{e}}+ \\
& \left(\frac{\partial c}{\partial \tilde{\mathbf{u}}_{r}}+\boldsymbol{\lambda}_{r}^{\mathrm{T}} \tilde{\mathbf{S}}_{r}-\boldsymbol{\lambda}_{i}^{\mathrm{T}} \tilde{\mathbf{S}}_{i}\right) \frac{\partial \tilde{\mathbf{u}}_{r}}{\partial \xi^{e}}+\left(\frac{\partial c}{\partial \tilde{\mathbf{u}}_{i}}-\boldsymbol{\lambda}_{i}^{\mathrm{T}} \tilde{\mathbf{S}}_{r}-\boldsymbol{\lambda}_{r}^{\mathrm{T}} \tilde{\mathbf{S}}_{i}\right) \frac{\partial \tilde{\mathbf{u}}_{i}}{\partial \xi^{e}} \\
& +\boldsymbol{\lambda}_{r}^{\mathrm{T}}\left(\frac{\partial \tilde{\mathbf{S}}_{r}}{\partial \xi^{e}} \tilde{\mathbf{u}}_{r}-\frac{\partial \tilde{\mathbf{S}}_{i}}{\partial \xi^{e}} \tilde{\mathbf{u}}_{i}\right)-\boldsymbol{\lambda}_{i}^{\mathrm{T}}\left(\frac{\partial \tilde{\mathbf{S}}_{i}}{\partial \xi^{e}} \tilde{\mathbf{u}}_{r}+\frac{\partial \tilde{\mathbf{S}}_{r}}{\partial \xi^{e}} \tilde{\mathbf{u}}_{i}\right)
\end{aligned}
$$

We will now choose the adjoint variable so that terms involving $\frac{\partial \tilde{\mathbf{u}}_{r}}{\partial \xi^{e}}$ and $\frac{\partial \tilde{\mathbf{u}}_{i}}{\partial \xi^{e}}$ vanish:

$$
\begin{array}{r}
\frac{\partial c}{\partial \tilde{\mathbf{u}}_{r}}+\boldsymbol{\lambda}_{r}^{\mathrm{T}} \tilde{\mathbf{S}}_{r}-\boldsymbol{\lambda}_{i}^{\mathrm{T}} \tilde{\mathbf{S}}_{i}=0 \\
\frac{\partial c}{\partial \tilde{\mathbf{u}}_{i}}-\boldsymbol{\lambda}_{i}^{\mathrm{T}} \tilde{\mathbf{S}}_{r}-\boldsymbol{\lambda}_{r}^{\mathrm{T}} \tilde{\mathbf{S}}_{i}=0
\end{array}
$$

which can be combined into:

$\boldsymbol{\lambda}^{\mathrm{T}} \tilde{\mathbf{S}}=-\left(\frac{\partial c}{\partial \tilde{\mathbf{u}}_{r}}-i \frac{\partial c}{\partial \tilde{\mathbf{u}}_{i}}\right)$ and after transposing the terms:

$$
\tilde{\mathbf{S}}^{\mathrm{T}} \boldsymbol{\lambda}=-\left(\frac{\partial c}{\partial \tilde{\mathbf{u}}_{r}}-i \frac{\partial c}{\partial \tilde{\mathbf{u}}_{i}}\right)^{\mathrm{T}}
$$

which can be solved to obtain $\boldsymbol{\lambda}$ whereafter the sensitivities can be obtained by rewriting Eq. (38) into

$\Phi^{\prime}=\frac{\partial c}{\partial \xi^{e}}+\operatorname{Re}\left(\lambda^{\mathrm{T}} \frac{\partial \tilde{\mathbf{S}}}{\partial \xi^{e}} \tilde{\mathbf{u}}\right)$

The term $\frac{\partial \tilde{\mathbf{S}}}{\partial \xi^{e}}$ in Eq. (42) can be written out as:

$\frac{\partial \tilde{\mathbf{S}}}{\partial \xi^{e}}=\frac{\partial \tilde{\mathbf{K}}}{\partial \xi^{e}}-\omega^{2} \frac{\partial \tilde{\mathbf{M}}}{\partial \xi^{e}}$

which we can specify further as

$$
\begin{gathered}
\frac{\partial \tilde{\mathbf{K}}}{\partial \xi^{e}}=\left[\begin{array}{cc}
\frac{\partial \mathbf{K}_{s}}{\partial \xi^{e}} & \frac{\partial \mathbf{S}_{p}}{\partial \xi^{e}} \\
\mathbf{0} & \frac{\partial \mathbf{K}_{a}}{\partial \xi^{e}}
\end{array}\right]=\left[\begin{array}{cc}
\frac{\partial E^{e}}{\partial \xi^{e}} \mathbf{K}_{s}^{e} & -\mathbf{S}^{e} \\
\mathbf{0} & \frac{\partial\left(\rho_{a}^{e}\right)^{-1}}{\partial \xi^{e}} \mathbf{K}_{a}^{e}
\end{array}\right] \\
\frac{\partial \tilde{\mathbf{M}}}{\partial \xi^{e}}=\left[\begin{array}{cc}
\frac{\partial \mathbf{M}_{s}}{\partial \xi^{e}} & \mathbf{0} \\
\frac{\partial \tilde{\mathbf{S}}_{u}}{\partial \xi^{e}} & \frac{\partial \mathbf{M}_{a}}{\partial \xi^{e}}
\end{array}\right]=\left[\begin{array}{cc}
\frac{\partial \rho_{s}^{e}}{\partial \xi^{e}} \mathbf{M}_{s}^{e} & \mathbf{0} \\
\left(\mathbf{S}^{e}\right)^{T} & \frac{\partial\left(\kappa^{e}\right)^{-1}}{\partial \xi^{e}} \mathbf{M}_{a}^{e}
\end{array}\right]
\end{gathered}
$$

The interpolation functions for the element material properties (acoustic and mechanical): $E^{e}, \rho_{a}^{e}, \rho_{s}^{e}$ and $\kappa^{e}$ will be specified below for the specific application example.

\subsection{Example}

As an example we consider a standard topology optimization problem for the setup depicted in Fig. 12. The slidingfixed beam is loaded symmetrically by two identical vertical time-harmonic forces $f \cos \omega t$ at the sliding support and is surrounded by an acoustic medium (air). The air domain has hard wall boundary conditions at the vertical boundaries and absorbing boundaries at the horizontal boundaries. The part of the domain occupied by the structure in the figure is used as design domain and may in the final design consist of structural and acoustic domains. The remaining part is a fixed acoustic domain. The geometrical, material and mesh parameters are listed in Tab. 3 .

We consider the following measure of dynamic compliance as our objective function:

$c=\left|\mathbf{f}_{s}^{\mathrm{T}} \mathbf{u}\right|=\left|\tilde{\mathbf{f}}^{\mathrm{T}} \tilde{\mathbf{u}}\right|=\sqrt{\left(\tilde{\mathbf{f}}^{\mathrm{T}} \tilde{\mathbf{u}}_{r}\right)^{2}+\left(\tilde{\mathbf{f}}^{\mathrm{T}} \tilde{\mathbf{u}}_{i}\right)^{2}}$

which is an extension of the minimum compliance design problem to the dynamic case. We consider only external structural loads and no acoustic sources and the objective function only includes the structural response. However, as will be shown the presence of the acoustic medium has an effect on the appearance of the optimized structure and the corresponding response. 


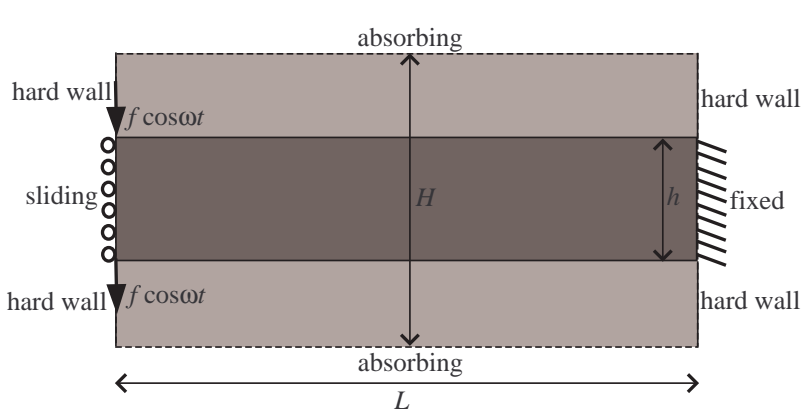

Fig. 12 A sliding-fixed beam subjected to time-harmonic structural loads and surrounded by an acoustic medium with open (absorbing) boundaries at top and bottom.

\begin{tabular}{|l|r|}
\hline$E$ & $500 \mathrm{MPa}$ \\
$\rho_{s}$ & $1200 \mathrm{~kg} / \mathrm{m}^{3}$ \\
$E$ (weak) & $25 \mathrm{MPa}$ \\
$\rho_{s}$ (weak) & $60 \mathrm{~kg} / \mathrm{m}^{3}$ \\
$v$ & 0.4 \\
$2 \mathrm{D}$ model & plane strain \\
$\rho_{a}$ & $1.204 \mathrm{~kg} / \mathrm{m}^{3}$ \\
$c$ & $342 \mathrm{~m} / \mathrm{s}$ \\
$H$ & $0.5 \mathrm{~m}$ \\
$h$ & $0.2 \mathrm{~m}$ \\
$L$ & $1.0 \mathrm{~m}$ \\
$\beta$ & 8 \\
mesh & $300 \times 150$ \\
\hline
\end{tabular}

Table 3 Geometrical, material and mesh parameters for the optimization example.

Eq. (41) for the adjoint problem specifically becomes:

$$
\begin{aligned}
\frac{\partial c}{\partial \tilde{\mathbf{u}}_{r}} & =\frac{1}{2 \sqrt{\left(\tilde{\mathbf{f}}^{\mathrm{T}} \tilde{\mathbf{u}}_{r}\right)^{2}+\left(\tilde{\mathbf{f}}^{\mathrm{T}} \tilde{\mathbf{u}}_{i}\right)^{2}}} 2\left(\tilde{\mathbf{f}}^{\mathrm{T}} \tilde{\mathbf{u}}_{r}\right) \tilde{\mathbf{f}}^{\mathrm{T}}=\frac{\left(\tilde{\mathbf{f}}^{\mathrm{T}} \tilde{\mathbf{u}}_{r}\right)^{\mathrm{T}}}{c} \\
\frac{\partial c}{\partial \tilde{\mathbf{u}}_{i}} & =\frac{1}{2 \sqrt{\left(\tilde{\mathbf{f}}^{\mathrm{T}} \tilde{\mathbf{u}}_{r}\right)^{2}+\left(\tilde{\mathbf{f}}^{\mathrm{T}} \tilde{\mathbf{u}}_{i}\right)^{2}}} 2\left(\tilde{\mathbf{f}}^{\mathrm{T}} \tilde{\mathbf{u}}_{i} \tilde{\mathbf{f}}^{\mathrm{T}}=\frac{\left(\tilde{\mathbf{f}}^{\mathrm{T}} \tilde{\mathbf{u}}_{i}\right) \tilde{\mathbf{f}}^{\mathrm{T}}}{c}\right. \\
\tilde{\mathbf{S}}^{\mathrm{T}} \boldsymbol{\lambda} & =-\left(\frac{\partial c}{\partial \tilde{\mathbf{u}}_{r}}-i \frac{\partial c}{\partial \tilde{\mathbf{u}}_{i}}\right)^{\mathrm{T}}=-\frac{\tilde{\mathbf{u}}^{*} \tilde{\mathbf{f}}_{\tilde{\mathbf{f}}}}{c}
\end{aligned}
$$

where $\tilde{\mathbf{u}}^{*}$ denotes the complex transpose of the state vector $\tilde{\mathbf{u}}$ and the sensitivities can then be found directly from Eq. (42) using that the objective function does not depend explicitly on the design variables so that $\partial c / \partial \xi^{e}=0$.

The interpolation functions for the material parameters are here chosen as:

$$
\begin{aligned}
E^{e} & =E_{v}+\frac{\xi^{e}}{1+q\left(1-\xi^{e}\right)}\left(E-E_{v}\right) \\
\rho_{s}^{e} & =\rho_{v}+\xi^{e}\left(\rho_{s}-\rho_{v}\right) \\
\frac{1}{\kappa^{e}} & =\frac{1}{\kappa}+\xi^{e}\left(\frac{1}{\kappa_{r}}-\frac{1}{\kappa}\right) \\
\frac{1}{\rho_{a}^{e}} & =\frac{1}{\rho_{a}}+\xi^{e}\left(\frac{1}{\rho_{r}}-\frac{1}{\rho_{a}}\right)
\end{aligned}
$$

i.e. a RAMP interpolation has been chosen for Young's modulus with $q$ as a penalization factor (Stolpe and Svanberg

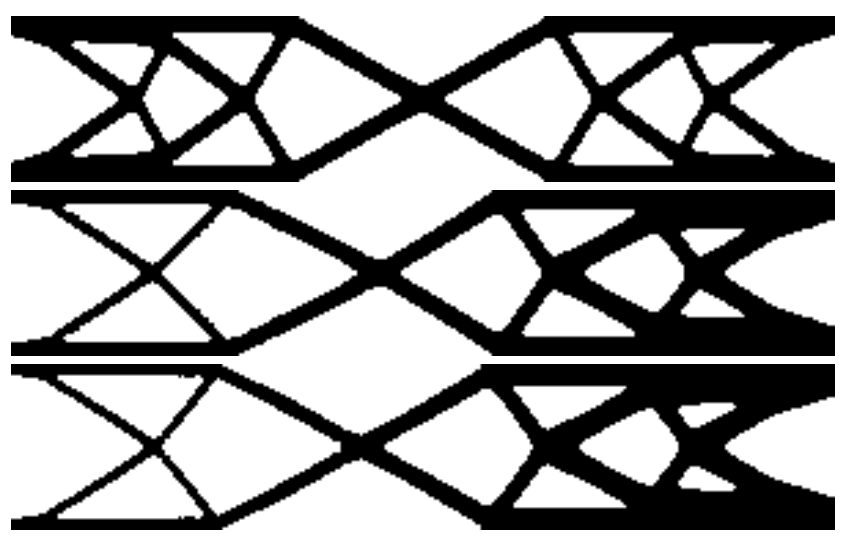

Fig. 13 Optimized designs for the design problem illustrated in Fig. 12. Top: static design, middle: dynamic design without acoustics, bottom: dynamic design with acoustics.

2001), combined with linear interpolation for the structural mass density. For the acoustic terms linear interpolations have been chosen for both $\frac{1}{\kappa^{e}}$ and $\frac{1}{\rho_{a}^{e}}$ in accordance with the standard procedure in topology optimization of pure acoustic problems (Christiansen et al 2015).

Thus the terms in Eqs.(44)-(45) can explicitly be calculated as:

$$
\begin{aligned}
\frac{\partial \rho_{s}^{e}}{\partial \xi^{e}} & =\rho_{s}-\rho_{v} \\
\frac{\partial\left(\kappa^{e}\right)^{-1}}{\partial \xi^{e}} & =\frac{1}{\kappa_{r}}-\frac{1}{\kappa} \\
\frac{\partial E^{e}}{\partial \xi^{e}} & =\frac{1+q}{1+q\left(1-\xi^{e}\right)^{2}}\left(E-E_{v}\right) \\
\frac{\partial\left(\rho_{a}^{e}\right)^{-1}}{\partial \xi^{e}} & =\frac{1}{\rho_{r}}-\frac{1}{\rho_{a}}
\end{aligned}
$$

The computed sensitivities are used along with the mathematical programming tool MMA (Svanberg 1987) in an iterative scheme to generate optimized designs. The implementation follows closely the outline in 88-line code provided by Andreassen et al (2011) using a density filter with a radius of 3.5 elements. A maximum of $40 \%$ of material is allowed to be used and the initial design is chosen as a homogeneous structure with $\xi^{e}=0.4$. We use a continuation scheme on the penalization parameter starting with the values of $q=0$ (linear stiffness interpolation), increasing to $q=1$ after 20 iterations and further increasing $q$ by 1 every 50 iterations. The procedure is terminated after a fixed number of iterations (250) and the optimized designs are thresholded to $0-1$ before the final analysis of the performance.

Initially, the optimization study is first carried out for a corresponding static problem using only the structural part of the system - ie. a normal static compliance minimization problem. The optimized structure is depicted in Fig. 13top. A second optimization problem is conducted without acoustic influence but for quasi-static conditions with a non-zero excitation frequency $(\omega=30 \times 2 \pi \mathrm{rad} / \mathrm{s})$ which is below the 
fundamental natural frequency of the initial structure. The optimized structure for the quasi-static condition is depicted in Fig. 13middle and is seen to differ from the structure optimized for static conditions. For static conditions zero moment is obtained at midpoint which is reflected in the optimized structure where only the transverse force needs to be transmitted and the vertical extent of material can be minimized to close-to a point (the finite extent is due to the use of the filter). When the inertial load is added, with a distribution close to the fundamental mode shape, the point of zero moment point is shifted to the left and the moment at the left boundary is reduced compared to the moment at the right boundary resulting in more material being shifted to the right part of the structure.

The problem is now solved with the full structural-acoustic interaction model. A different set of material parameters is introduced corresponding to a very weak and light material with both density and Young's modulus reduced by a factor of 20. The two material sets have identical stiffnessto-mass ratios and therefore both static and quasi-static designs are independent of which material set is used. However, the acoustic-mechanical coupling strength inherently depends on material impedance given as $\sqrt{E_{s} \rho_{s}}$. For the normal material the coupling is weak enough so that the optimized structure has an appearance very similar to the quasi-static design (Fig. 13middle). However, for the artificial weak material the zero moment point is now shifted further to the left due to the added pressure load from the acoustic domain. This is seen in the optimized structure shown in Fig. 13bottom, where it is also noted that even more material is shifted from the location near the left boundary to the region near the right boundary where the moment has increased further.

Fig. 14 illustrates the evolution of the acoustic-structural interaction during the optimization procedure. The acoustically induced load vector is plotted for the initial material configuration, an intermediate design step and close to final convergence of the optimization scheme. For the initial homogenous material distribution the acoustic loading is confined to the upper and lower structural boundaries whereas the internal loads effectively cancel out. The intermediate design step possesses inhomogeneous material distribution including elements with intermediate values of the design variable. As appears, this induces distributed acoustic loads throughout the structure. It should here be noted that a nonlinear scaling of the arrow sizes has been applied in order to also properly visualize forces with smaller magnitude and for clarity not all nodal values are shown. In the almost final design with a close to black-white distribution it is again seen how the induced loads affect mainly the structural boundaries. Furthermore, it can be seen that the forces are largest on the outermost boundaries indicating that induced acoustic pressure in the internal structural cavities is rela-
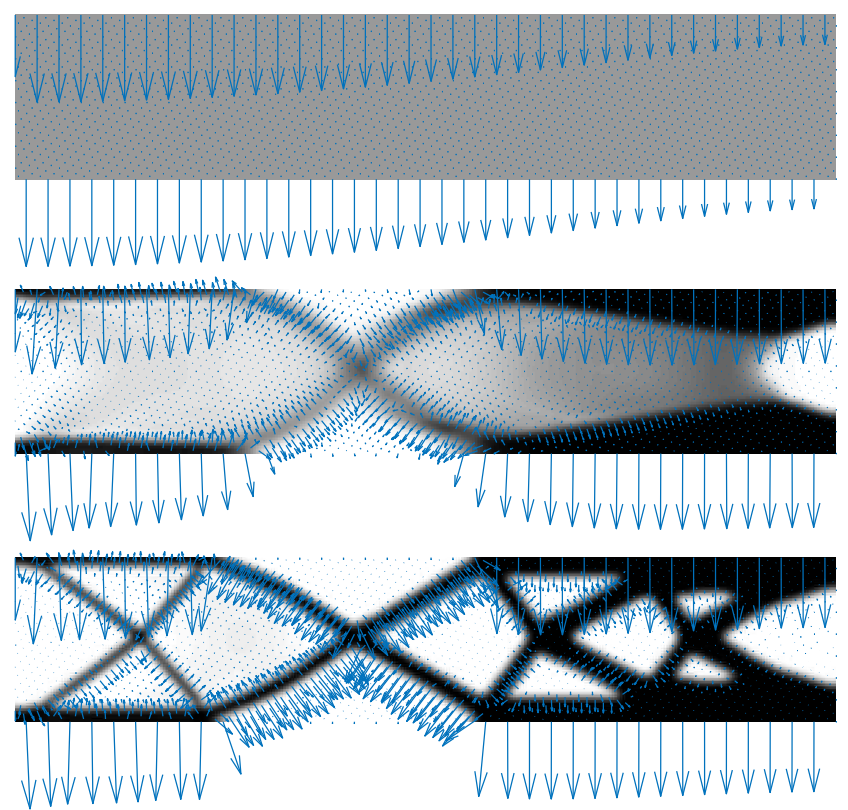

Fig. 14 Acoustically induced structural loads for top: initial material distribution, middle: intermediate design step and bottom: close to final design.

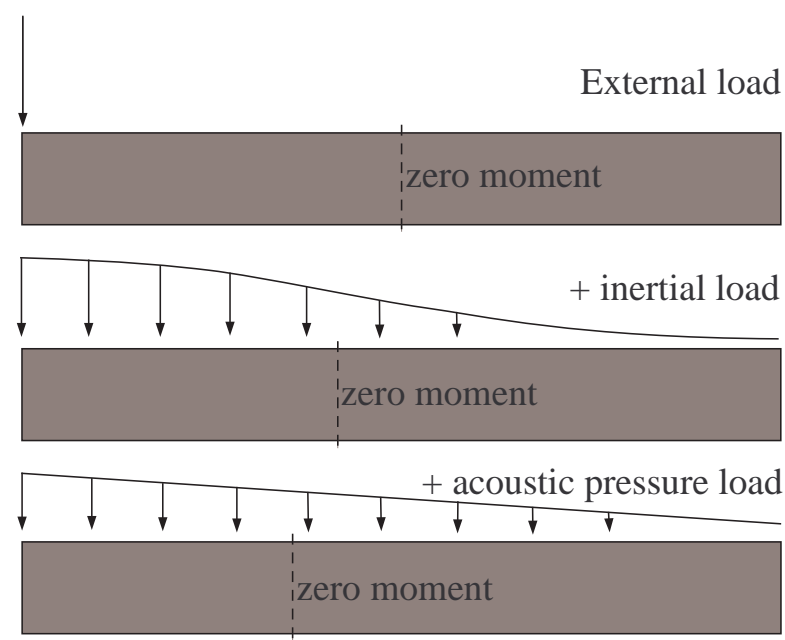

Fig. 15 Illustration of the effect of the dynamic and acoustic loads on the structure and indication of the point of zero moment.

tively small. The evolution of structurally induced acoustic loads follows a similar pattern and is not shown.

In Fig. 15 the moment and load considerations are further outlined giving a schematic illustration of the loads appearing for the three conditions. It should be emphasized that the acoustic pressure distribution is assumed here to follow a linear distribution which is clearly an approximation. However the precise distribution does not change the conclusion.

In Fig. 16 we show the frequency response of the three optimized structures with all simulations done using the full 


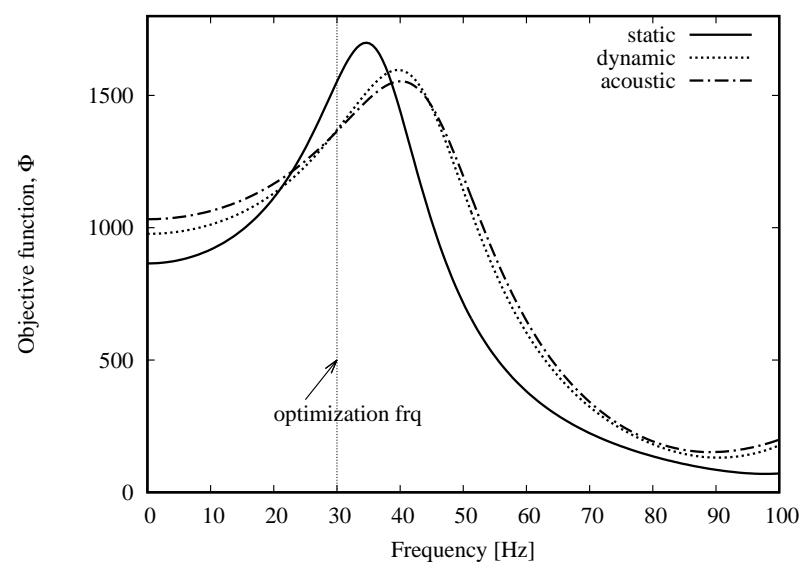

Fig. 16 Frequency response for the three optimized designs for artificial weak material.

acoustic-structure interaction model for the artificial light and flexible material. It is noted that the design optimized for acoustic coupling conditions performs better than the other optimized designs although the quasi-static design has only a slightly higher compliance at the optimization frequency.

It should be mentioned that the computational effort required to solve the coupled state problem during the optimization procedure with intermediate design variables generally increases due the populated coupling matrices that reduces sparsity. For the presented example an increase up to a factor of two was observed compared to the case of a 0-1 discrete design.

\section{Summary and conclusions}

A simple computational scheme for acoustic-structure interaction simulation has been proposed. The computational framework for the method has been presented in detail including examples to illustrate the basic properties and limitations of the method. Furthermore, the application of the method for acoustic-structural topology optimization has been demonstrated.

The method is based on extended finite element models of both structural vibrations and acoustic pressure fluctuations in the entire modelling domain. The individual domains are identified using an element topological indicator function and the non-structural and non-acoustic parts of the domain are assigned artificial structural and acoustic material parameters representing structural void and an acoustically rigid material, respectively. By choosing appropriate contrast between physical and artificial material parameters the accuracy of the models can be controlled efficiently which is illustrated by an simple example involving uncoupled structural and acoustic eigenvalues.

The coupling between the acoustic pressure fluctuations and the structural vibration takes the form of structural bound- ary loads from the acoustic pressure and acoustic sources from the vibrating structural boundary. The proposed scheme circumvents the need for explicit tracking of the interface boundaries. Instead an self-coupling element matrix is defined which is assembled over the entire modelling domain to produce the desired loads and sources at the interface. The performance of the full coupled model is demonstrated for the case of transmission of a plane acoustic wave through a flexible partition. The proposed scheme shows excellent accuracy when compared to a similar COMSOL model provided that the contrast between the physical and artificial material parameters is sufficiently high. If a too low contrast is used the model suffers inaccuracies due to acoustic leakage through the non-acoustic domain.

In the final part of the paper the applicability to topology optimization problems is demonstrated. The discrete topological indicator function introduced in the first part of the paper is replaced by a continuous indicator function. In this way the coupling between the two physical fields is active on the entire domain where the indicator function is not strictly 0 or 1 . The use of the continuous indicator function allows for computing the gradient of a general objective function directly and facilitates the use of a standard mathematical programming tool for design optimization. The use of the algorithm is demonstrated for dynamic minimum compliance design of a beam with a time-harmonic structural load embedded in a semi-open acoustic domain. The generated design are compared to design without the acoustic domain included and the observed differences is explained using simple physical considerations.

The major strength of the proposed method lies in the simplicity of implementation using standard FE formulation for structural and acoustic problems on a fixed mesh and the straightforward assembly of the coupling matrices using the topological indicator function. The major drawback is the required computational effort due to the need for solving the acoustic and structural problems in the entire domain, however, this is also the case for methods based on the mixed formulation and the UMP approach. Additionally, the loss of sparsity due the populated coupling matrices also causes an increase in computational efforts. Additionally, to broaden the general applicability of the proposed method, further work could be done in refining the choice of interpolation and contrast models for both the acoustic and structural problem.

\section{Appendix}

The computation of $\mathbf{S}^{e}$ is exemplified for the bilinear 4noded rectangular element (Q4) for both the structural and acoustic domain. It should be emphasized that it is troublefree to use different approximation-orders for the acoustic 


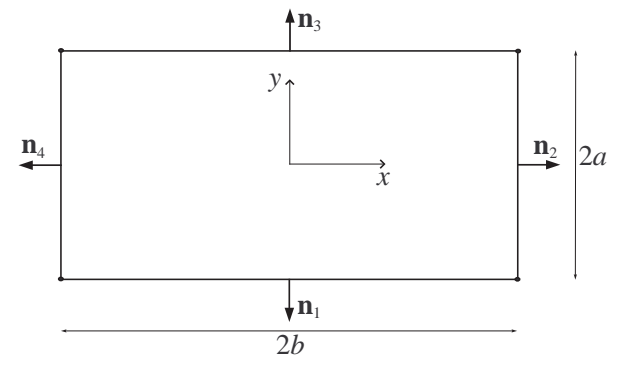

Fig. 17 Basic element geometry for the 4-node rectangular element.

and structural parts. The basic element geometry is shown in Fig. 17.

Specifically the shape functions are defined as:

$\mathbf{N}_{a}=\frac{1}{4 a b}\left[\begin{array}{llll}N_{1} & N_{2} & N_{3} & N_{4}\end{array}\right]$

and

$\mathbf{N}_{s}=\frac{1}{4 a b}\left[\begin{array}{cccccccc}N_{1} & 0 & N_{2} & 0 & N_{3} & 0 & N_{4} & 0 \\ 0 & N_{1} & 0 & N_{2} & 0 & N_{3} & 0 & N_{4}\end{array}\right]$

where

$N_{1}=\frac{1}{4 a b}(a-x)(b-y)$

$N_{2}=\frac{1}{4 a b}(a+x)(b-y)$

$N_{3}=\frac{1}{4 a b}(a+x)(b+y)$

$N_{4}=\frac{1}{4 a b}(a-x)(b+y)$

and the computation of the self-interaction matrix can be written out as:

$$
\begin{aligned}
\mathbf{S}^{e} & =\left.\int_{-a}^{a}\left(\mathbf{n}_{1}^{T} \mathbf{N}_{s}\right)^{T} \mathbf{N}_{a}\right|_{y=-b} \mathrm{~d} x+\left.\int_{-b}^{b}\left(\mathbf{n}_{2}^{T} \mathbf{N}_{s}\right)^{T} \mathbf{N}_{a}\right|_{x=a} \mathrm{~d} y \\
& +\left.\int_{-a}^{a}\left(\mathbf{n}_{3}^{T} \mathbf{N}_{s}\right)^{T} \mathbf{N}_{a}\right|_{y=b} \mathrm{~d} x+\left.\int_{-b}^{b}\left(\mathbf{n}_{4}^{T} \mathbf{N}_{s}\right)^{T} \mathbf{N}_{a}\right|_{x=-a} \mathrm{~d} y
\end{aligned}
$$

which results in the following matrix:

$\mathbf{S}^{e}=\frac{1}{3}\left[\begin{array}{cccc}-2 b & 0 & 0 & -b \\ -2 a & -a & 0 & 0 \\ 0 & 2 b & b & 0 \\ -a & -2 a & 0 & 0 \\ 0 & b & 2 b & 0 \\ 0 & 0 & 2 a & a \\ -b & 0 & 0 & -2 b \\ 0 & 0 & a & 2 a\end{array}\right]$

\section{References}

Akl W, El-Sabbagh A, Al-Mitani K, Baz A (2009) Topology optimization of a plate coupled with acoustic cavity. International Journal of Solids and Structures 46(10):20602074
Allaire G, Jouve F, Toader A (2002) A level-set method for shape optimization. Comptes Rendus Mathematique 334(12):1125-1130

Andreassen E, Clausen A, Schevenels M, Lazarov BS, Sigmund O (2011) Efficient topology optimization in matlab using 88 lines of code. Struct Multidisc Optim 43(1):1-16

Bendsøe M, Sigmund O (2003) Topology Optimization. Theory, Methods and Applications. Springer

Chen N, Yu D, Xia B, Liu J, Ma Z (2017) Microstructural topology optimization of structural-acoustic coupled systems for minimizing sound pressure level. Structural and Multidisciplinary Optimization 56(6):1259-1270

Christiansen RE, Lazarov BS, Jensen JS, Sigmund O (2015) Creating geometrically robust designs for highly sensitive problems using topology optimization. Structural and Multidisciplinary Optimization 52(4):737-754

Cook RD, Malkus DS, Plesha ME, Witt RJ (2002) Concepts and Applications of Finite Element Analysis, 4th edn. John Wiley and Sons

Desai J, Faure A, Michailidis G, Parry G, Estevez R (2018) Topology optimization in acoustics and elasto-acoustics via a level-set method. Journal of Sound and Vibration 420:73-103

Du J, Olhoff N (2010) Topological design of vibrating structures with respect to optimum sound pressure characteristics in a surrounding acoustic medium. Structural and Multidisciplinary Optimization 42(1):43-54

Kasolis F, Wadbro E, Berggren M (2015) Analysis of fictitious domain approximations of hard scatterers. Siam Journal on Numerical Analysis 53(5):2347-2362, DOI $10.1137 / 140981630$

Kook J (2017) Evolutionary topology optimization for acoustic-structure interaction problems using a mixed $\mathrm{u} / \mathrm{p}$ formulation. Submitted

Kook J, Jensen JS (2017) Topology optimization of periodic microstructures for enhanced loss factor using acousticstructure interaction. International Journal of Solids and Structures 122:59-68

Lee JS, Kang YJ, Kim YY (2012) Unified multiphase modeling for evolving, acoustically coupled systems consisting of acoustic, elastic, poroelastic media and septa. Journal of Sound and Vibration 331(25):5518 - 5536

Lee JS, Göransson P, Kim YY (2015) Topology optimization for three-phase materials distribution in a dissipative expansion chamber by unified multiphase modeling approach. Computer Methods in Applied Mechanics and Engineering 287:191 - 211

Marburg S (2002) Developments in structural-acoustic optimization for passive noise control. Archives of Computational Methods in Engineering 9(4):291-370

Miyata K, Noguchi Y, Yamada T, Izui K, Nishiwaki S (2018) Optimum design of a multi-functional acoustic metasurface using topology optimization based on zwicker's 
loudness model. Computer Methods in Applied Mechanics and Engineering 331:116 - 137

Noguchi Y, Yamada T, Otomori M, Izui K, Nishiwaki S (2015) An acoustic metasurface design for wave motion conversion of longitudinal waves to transverse waves using topology optimization. Applied Physics Letters 107(22):221,909

Noguchi Y, Yamada T, Yamamoto T, Izui K, Nishiwaki S (2016) Topological derivative for an acoustic-elastic coupled system based on two-phase material model. Mechanical Engineering Letters 2:16-00,246-16-00,246

Noguchi Y, Yamamoto T, Yamada T, Izui K, Nishiwaki S (2017) A level set-based topology optimization method for simultaneous design of elastic structure and coupled acoustic cavity using a two-phase material model. Journal of Sound and Vibration 404:15-30

Osher S, Santosa F (2001) Level set methods for optimization problems involving geometry and constraints I. Frequencies of a two-density inhomogeneous drum. Journal of Computational Physics 171(1):272-288

Osher S, Sethian J (1988) Fronts propagating with curvature-dependent speed - algorithms based on Hamilton-Jacobi formulations. Journal of Computational Physics 79(1):12-49

Sethian J, Wiegmann A (2000) Structural boundary design via level set and immersed interface methods. Journal of Computational Physics 163(2):489-528

Shu L, Wang MY, Ma Z (2014) Level set based topology optimization of vibrating structures for coupled acousticstructural dynamics. Computers \& Structures 132:34-42

Sigmund O, Clausen PM (2007) Topology optimization using a mixed formulation: An alternative way to solve pressure load problems. Computer Methods in Applied Mechanics and Engineering 196(13-16):1874-1889

Søndergaard MB, Pedersen CB (2014) Applied topology optimization of vibro-acoustic hearing instrument models. Journal of Sound and Vibration 333(3):683 - 692

Stolpe M, Svanberg K (2001) An alternative interpolation scheme for minimum compliance topology optimization. Structural and Multidisciplinary Optimization 22(2):116124

Svanberg K (1987) Method of moving asymptotes - a new method for structural optimization. Int J Numer Meth Eng 24(2):359-373

Vicente W, Picelli R, Pavanello R, Xie Y (2015) Topology optimization of frequency responses of fluid-structure interaction systems. Finite Elements in Analysis and Design 98(Supplement C):1 - 13

Wang M, Wang X, Guo D (2003) A level set method for structural topology optimization. Computer Methods in Applied Mechanics and Engineering 192(1-2):227-246

Wang X, Bathe K (1997) Displacement pressure based mixed finite element formulations for acoustic fluid- structure interaction problems. International Journal for Numerical Methods in Engineering 40(11):2001-2017

Yoon GH, Sigmund O (2008) A monolithic approach for topology optimization of electrostatically actuated devices. Computer Methods in Applied Mechanics and Engineering 197(45-48):4062-4075

Yoon GH, Jensen JS, Sigmund O (2007) Topology optimization of acoustic-structure interaction problems using a mixed finite element formulation. International Journal for Numerical Methods in Engineering 70(9):1049-1075 